\title{
AN UPPER BOUND FOR THE WAITING TIME FOR NONLINEAR DEGENERATE PARABOLIC EQUATIONS
}

\author{
BY
}

MICHEL CHIPOT AND THOMAS SIDERIS ${ }^{1}$

\begin{abstract}
An upper bound is obtained for the time when the support of the solution of some nonlinear, degenerate parabolic equations begins to spread.
\end{abstract}

Solutions to many nonlinear degenerate diffusion equations which are compactly supported initially, remain so at future times (see $[\mathbf{4}, \mathbf{7}, \mathbf{8}]$ ). We obtain here an upper bound for the time when the support begins to spread, the so-called waiting time. In one space dimension such an upper bound was found by B. F. Knerr [7] who derived a weak ordinary differential equation along the interface (the boundary of the support) and used it to show that the waiting time depends on the size of the initial data near the interface (see also $[\mathbf{2}, \mathbf{4}, \mathbf{1 1}, \mathbf{1 2}]$ ).

We replace the equation along the interface with a first order differential inequality for a local average of the solution near a point on the interface. This allows estimation of the waiting time in higher dimensions, while in one dimension it reproduces previous results $[\mathbf{4}, \mathbf{7}]$.

In the particular case of the porous medium equation, similar results can also be obtained from a new and sharp Harnack inequality due to D. G. Aronson and L. A. Caffarelli $[\mathbf{3}]$. N. D. Alikakos [1] has recently supplied a necessary and sufficient condition for zero waiting time in higher dimensions which relies, in part, upon this inequality. Nevertheless, even for the case of the porous medium equation, our results are elementary and self-contained.

Finally, we would like to thank J. L. Vasquez for an informative conversation with the second author and for suggesting that we seek a local version of our preliminary results.

1. Preliminaries. Let $u_{0}$ and $g$ be nonnegative functions.

A function $u(x, t)$ is said to be a weak solution of the nonlinear degenerate parabolic equation $u_{t}=\Delta g(u), u(x, 0)=u_{0}(x)$ if, for some $T>0, u$ satisfies:

(i) $u$ is continuous on $\mathbf{R}^{n} \times(0, T)$,

(ii) $u \geq 0$,

(iii) $\int_{0}^{T} \int_{\mathbf{R}^{n}}\left[u \varphi_{t}+g(u) \Delta \varphi\right] d x d t+\int_{\mathbf{R}^{n}} u_{0}(x) \varphi(x, 0) d x=0$

$\forall \varphi \in H^{2}\left(\mathbf{R}^{n} \times(0, T)\right)$ with compact support in $\mathbf{R}^{n} \times[0, T)$.

Received by the editors September 19, 1983 and, in revised form, May 18, 1984.

1980 Mathematics Subject Classification. Primary 35K65, 35B99.

Key words and phrases. Nonlinear degenerate parabolic equations, waiting time.

${ }^{1}$ Supported in part by the National Science Foundation.

(C) 1985 American Mathematical Society $0002-9947 / 85 \$ 1.00+\$ .25$ per page 
Under appropriate restrictions on $u_{0}$ and $g$, there exists a unique solution of $(\mathrm{P})$ (see for example [5-11]). For our results we need only assume

$$
g(u) \geq C \cdot u^{m} \text { for all } u \geq 0
$$

( $m$ and $C$ are strictly positive constants, $m>1$ ).

We wish to study how the support of $u(\cdot, t)$ spreads with time. More precisely, if $\Omega$ is an open set in $\mathbf{R}^{n}$ such that $u_{0}(x)=0 \forall x \in \mathbf{R}^{n} \backslash \Omega$ let us denote the waiting time for $(\mathrm{P})$ associated with $\Omega$ to be

$$
t_{\Omega}^{*}=\operatorname{Sup}\left\{t \geq 0: u(x, t)=0 \forall x \in \mathbf{R}^{n} \backslash \Omega\right\} .
$$

We will derive an upper bound for $t_{\Omega}^{*}$ which depends on the behavior of $u_{0}$ in a neighborhood of a point in $\partial \Omega$. This bound will be related to the life span for solutions to nonlinear differential inequality.

First we prepare two technical lemmias.

Fix $x_{0} \in \mathbf{R}^{n}$ and $\varepsilon>0$. We define the $H^{2}$ function

$$
h(x)=\left[\left(\varepsilon^{2}-\left|x-x_{0}\right|^{2}\right)^{+}\right]^{2},
$$

where ()$^{+}$denotes the positive part of a function and || the usual Euclidean norm in $\mathbf{R}^{n}$.

Then we have

LEMMA 1. If $((n+1) /(n+2)) \varepsilon^{2} \leq\left|x-x_{0}\right|^{2}$, then $\Delta h(x) \geq \varepsilon^{-4 m+2} h(x)^{m}$ for any $m>1$.

PROOF. It suffices to prove this for $x_{0}=0$ and

$$
\frac{n+1}{n+2} \cdot \varepsilon^{2} \leq|x|^{2}=r^{2}<\varepsilon^{2}
$$

So, we compute

$$
\begin{aligned}
\Delta h(x) & =h^{\prime \prime}(r)+\frac{n-1}{r} h^{\prime}(r) \\
& =4\left[(n+2) r^{2}-n \varepsilon^{2}\right] \geq 4 \varepsilon^{2} \\
& \geq \varepsilon^{-4 m+2} h(x)^{m},
\end{aligned}
$$

since $\varepsilon^{-2}\left(\varepsilon^{2}-r^{2}\right) \leq 1$.

Lemma 2. Let $A, B, m>1$ be positive constants. If $F(\tau)$ is a positive $C^{1}$ function on $\left(0, t^{*}\right)$ satisfying $F(0)=0$ and

$$
F^{\prime}(\tau) \geq A \tau^{2}+B \tau^{-3(m-1)} F(\tau)^{m}
$$

then $t^{*} \leq C / A^{m-1} \cdot B$, where the constant $C$ depends only on $m$.

ProOF. We may assume $t^{*}>0$. First, from $F^{\prime}(\tau) \geq A \tau^{2}$ we deduce that

$$
F(\tau) \geq \frac{A}{3} \tau^{3}
$$

since $F(0)=0$. Choose $0<\nu<\frac{1}{3}, \nu<m-1$ and use (2) and (3); we obtain

$$
\begin{aligned}
F^{\prime}(\tau) & \geq B \tau^{-3(m-1)} F(\tau)^{1+\nu} \cdot F(\tau)^{m-1-\nu} \\
& \geq C A^{m-1-\nu} B \cdot \tau^{-3 \nu} F(\tau)^{1+\nu} .
\end{aligned}
$$


Or, since $F(\tau)>0$,

$$
F^{-1-\nu}(\tau) F^{\prime}(\tau) \geq C A^{m-1-\nu} B \tau^{-3 \nu}
$$

Integrating over $\left(\alpha t^{*}, t^{*}\right)$ for $0<\alpha<1$, we obtain

$$
\frac{F\left(\alpha t^{*}\right)^{-\nu}}{\nu} \geq \frac{1}{\nu}\left[F\left(\alpha t^{*}\right)^{-\nu}-F\left(t^{*}\right)^{-\nu}\right] \geq C A^{m-1-\nu} B\left(t^{*}\right)^{1-3 \nu}\left(1-\alpha^{1-3 \nu}\right) .
$$

Using (3) again leads to

$$
\nu^{-1}\left[\frac{A}{3}\left(\alpha t^{*}\right)^{3}\right]^{-\nu} \geq C A^{m-1-\nu} B\left(t^{*}\right)^{1-3 \nu}\left(1-\alpha^{1-3 \nu}\right)
$$

or, equivalently, $t^{*} \leq C / A^{m-1} B$.

2. The upper bound. We are now ready to state our result. Recall that $\Omega$ is an open set outside of which $u_{0} \equiv 0$. Fix $x_{0} \notin \bar{\Omega}$ and let $\delta$ denote the (positive) distance of $x_{0}$ from $\Omega$. For any $\varepsilon>0$, let us define

$$
C\left(u_{0} ; x_{0}, \varepsilon\right)=\int_{\Omega_{\varepsilon}} u_{0}(x) h(x) d x
$$

where $h$ is as in Lemma 1 and $\Omega_{\varepsilon}=\Omega \cap B_{\varepsilon}\left(x_{0}\right)$. (Here, $B_{\varepsilon}\left(x_{0}\right)=\left\{\left|x-x_{0}\right|<\varepsilon\right\}$.)

If $u$ is assumed to be a solution of $(\mathrm{P})$ our main result is

THEOREM. If $t_{\Omega}^{*}$ is the waiting time associated with $\Omega$, then

$$
t_{\Omega}^{*} \leq C \cdot \operatorname{Inf}_{x_{0}, \varepsilon}\left\{\left[\frac{\varepsilon^{4+2 /(m-1)}\left|\Omega_{\varepsilon}\right|}{C\left(u_{0} ; x_{0}, \varepsilon\right)}\right]^{m-1}: \frac{n+1}{n+2} \varepsilon^{2} \leq \delta^{2}\right\},
$$

where $C$ depends only on $m$ and the constant in $(1) .(|\cdot|$ denotes Lebesgue measure.)

ProOF. We may assume that $t_{\Omega}^{*}>0$, so for $0<\tau<t_{\Omega}^{*}$ we define the test function $\varphi(x, t)=\left[(\tau-t)^{+}\right]^{2} h(x)$, where $h$ is as in Lemma 1 (other choices are possible!). Noting that $u(x, t) \equiv 0$ outside of $\Omega$ when $t<t_{\Omega}^{*}$, we have from (P)(iii), using the above test function,

$$
\begin{aligned}
0= & -2 \int_{0}^{\tau} \int_{\Omega_{\varepsilon}}(\tau-t) h(x) u(x, t) d x d t \\
& +\int_{0}^{\tau} \int_{\Omega_{\varepsilon}}(\tau-t)^{2} \Delta h(x) g(u(x, t)) d x d t+C\left(u_{0} ; x_{0}, \varepsilon\right) \tau^{2} .
\end{aligned}
$$

Now, if we set

$$
F(\tau)=\int_{0}^{\tau} \int_{\Omega_{\varepsilon}}(\tau-t)^{2} h(x) u(x, t) d x d t
$$

then $F$ is $\left(?^{\circ}\right.$ on $\left(0, l_{52}^{*}\right)$, and

$$
F^{\prime}(\tau)=2 \int_{0}^{\tau} \int_{\Omega_{\varepsilon}}(\tau-t) h(x) u(x, t) d x d t .
$$

Note that $\Omega_{\varepsilon} \subset\left\{\left|x-x_{0}\right|^{2} \geq((n+1) /(n+2)) \varepsilon^{2}\right\}$ since $((n+1) /(n+2)) \varepsilon^{2} \leq \delta^{2}$.

So by $(4),(5),(P)(i i)$, Lemma 1 and (1) we deduce

$$
F^{\prime}(\tau) \geq C\left(u_{0} ; x_{0}, \varepsilon\right) \tau^{2}+C \varepsilon^{-4 m+2} \int_{0}^{\tau} \int_{\Omega_{\varepsilon}}(\tau-t)^{2} h^{m}(x) u^{m}(x, t) d x d t
$$


An application of Hölder's inequality with respect to the measure $d \mu=$ $(\tau-t)^{2} d x d t$ on $\Omega_{\varepsilon} \times(0, \tau)$ shows that

$$
\begin{aligned}
F(\tau) & =\int_{0}^{\tau} \int_{\Omega_{\varepsilon}} h(x) u(x, t) d \mu \\
& \leq\left(\int_{0}^{\tau} \int_{\Omega_{\varepsilon}} h^{m}(x) u^{m}(x, t) d \mu\right)^{1 / m}\left(\int_{0}^{\tau} \int_{\Omega_{\varepsilon}} d \mu\right)^{(m-1) / m} .
\end{aligned}
$$

Using the fact that

$$
\int_{0}^{\tau} \int_{\Omega_{\varepsilon}} d \mu=\int_{0}^{\tau} \int_{\Omega_{\varepsilon}}(\tau-t)^{2} d x d t=\left|\Omega_{\varepsilon}\right| \cdot \frac{\tau^{3}}{3}
$$

we obtain from $(7)$

$$
\int_{0}^{\tau} \int_{\Omega_{\varepsilon}}(\tau-t)^{2} h^{m}(x) u^{m}(x, t) d x d t \geq\left[\frac{3}{\tau^{3}\left|\Omega_{\varepsilon}\right|}\right]^{m-1} F(\tau)^{m}
$$

Combining (6) and (8) and then using Lemma 2 completes the proof.

We remark that if the infimum in the statement of the theorem is zero, then $t_{\Omega}^{*}=0$, so that the support of the solution $u$ of $(\mathrm{P})$ beings to spread immediately.

As an example of our result, let us examine the behavior of $t_{\Omega}^{*}$ in the following situation. Assume that for $y \in \partial \Omega$

$$
u_{0}(x) \geq \alpha|x-y|^{\gamma}+o\left(|x-t|^{\gamma}\right) \text { on } \varepsilon,
$$

where $\mathcal{C}$ is a small cone in $\Omega$ with vertex $y$ (see the figure) and $\alpha, \gamma$ are positive constants with $\gamma \leq 2 /(m-1)$. (When $n=1$, $\varepsilon$ is taken to be an interval.)

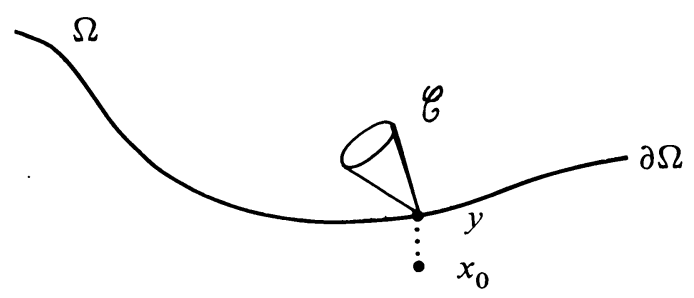

Choose $x_{0}$ such that $\left|x_{0}-y\right|=\operatorname{dist}\left(x_{0}, \Omega\right)=\delta$ and set $\varepsilon=((n+2) /(n+1))^{1 / 2} \delta$.

We then have

$$
\begin{aligned}
C\left(u_{0} ; x_{0}, \varepsilon\right) & \geq \int_{\varepsilon \cap B_{\varepsilon}\left(x_{0}\right)} u_{0}(x)\left(\varepsilon^{2}-\left|x-x_{0}\right|^{2}\right)^{2} d x \\
& \geq k \alpha \delta^{4+\gamma+n}+o\left(\delta^{4+\gamma+n}\right),
\end{aligned}
$$

where $k$ (and constants hereafter) depend only on $n$ and $\varepsilon$.

Thus, we deduce (since $\left|\Omega_{\varepsilon}\right|<C \varepsilon^{n}$ )

$$
\frac{\varepsilon^{4+2 /(m-1)}\left|\Omega_{\varepsilon}\right|}{C\left(u_{0} ; x_{0}, \varepsilon\right)} \leq C \frac{\delta^{4+2 /(m-1)+n}}{\alpha \delta^{4+\gamma+n}+o\left(\delta^{4+\gamma+n}\right)} .
$$

If $\gamma<2 /(m-1)$, then taking the infimum over $\delta>0$, we see that $t_{\Omega}^{*}=0$. When $n=1$, this is a result of $[\mathbf{7}]$.

On the other hand, if $\gamma=2 /(m-1)$ we obtain $t_{\Omega}^{*} \leq C / \alpha^{m-1}$. In the case $n=1$, this upper bound was obtained in [4] (for the porous medium equation), but there the constant is sharp. 
These examples show that, roughly speaking, the more dense the gas, directly behind the interface, the sooner the interface will spread.

We could just as well have defined a local waiting time, i.e.,

$$
t_{\Omega, B}^{*}=\sup \left\{t \geq 0: u(x, t)=0, \text { for } x \in\left(R^{n} \backslash \Omega\right) \cap B\right\},
$$

where $B$ is a ball centered on $\partial \Omega$. Our method also provides an upper bound for $t_{\Omega, B}^{*}$, since our test functions $h$ are local.

We also point out that the results carry over to the case where the Laplacian is replaced by a strongly elliptic operator in divergence form. (Lemma 1 holds with minor changes.) Finally, we could allow the appropriate inequality in (P)(iii).

\section{REFERENCES}

1. N. D. Alikakos, On the pointwise behavior of the solutions of the porous medium equation as $t$ approaches zero or infinity (to appear).

2. D. G. Aronson, Some properties of the interface for gas flow in porous media, Proceedings of the Montecatini Symposium on Free Boundary Problems, Pitman, New York, 1983.

3. D. G. Aronson and L. A. Caffarelli, The initial trace of a solution of the porous medium equations (to appear).

4. D. G. Aronson, L. A. Caffarelli and S. Kamin, How an initially stationary interface begins to move in porous medium flow, SIAM J. Math. Anal. 14 (1983), 639-658.

5. H. Brezis and M. G. Crandall, Uniqueness of solution of the initial-value problem for $u_{t}-\Delta \phi(u)=0$, J. Math. Pures Appl. (9) 58 (1979), 153-163.

6. L. A. Caffarelli and A. Friedman, Continuity of the density of a gas flow in a porous medium, Trans. Amer. Math. Soc. 252 (1979), 99-111.

7. B. F. Knerr, The porous medium equation in one dimension, Trans. Amer. Math. Soc. 234 (1977) $381-415$.

8. O. A. Oleinik, A. S. Kalasnikov and Yui-lin'Czou, The Cauchy problem and boundary problems for equations of the type of nonstationary fultration, Izv. Akad. Nauk SSSR Ser. Mat. 22 (1958), $607-704$.

9. L. A. Peletier, The porous media equation, Applications of Nonlinear Analysis in Physical Sciences (Proceedings of a meeting held at Bielefeld, W. Germany, 1979), Pitman, New York, 1981.

10. E. S. Sabinina, On the Cauchy problem for the equations of nonstationary gas filtration in several space variables, Dokl. Akad. Nauk SSSR 136 (1961), 1034-1037.

11. J. L. Vazquez, Asymptotic behaviour and propagation properties of the one-dimensional flow of gas in a porous medium, Trans. Amer. Math. Soc. 277 (1983), 506-527.

12. _ The interfaces of one-dimensional flows in porous media (to appear).

Université de Nancy I, U.E.R. SCiences Mathématiques, BP 239-54506 VanDOEUVRE CEDEX, FranCE

INSTITUTE FOR MATHEMATICS AND ITS APPLICATIONS, UNIVERSITY OF MiNNESOTA, MinNEAPOLIS, MiNnESOTA 55455 (Current address of M. Chipot)

Department of Mathematics, University of California, Santa Barbara, CALIFORNIA 93106 (Current address of T. Sideris) 